\title{
Stochastic Simulation of Scalar Mixing Capturing Unsteadiness and Small-scale Structure Based on Mean-flow Properties
}

\author{
Sigurd Sannan · Torleif Weydahl • Alan R. Kerstein
}

Received: 22 December 2011 / Accepted: 16 November 2012 / Published online: 11 December 2012

(C) The Author(s) 2012. This article is published with open access at Springerlink.com

\begin{abstract}
A key limitation of Reynolds-Average Navier-Stokes (RANS) simulation of mixing and reaction in turbulent flows is the lack of resolution of small-scale structure and associated unsteadiness. Various subgrid models formulated in state space have been developed to complement the RANS solution in this regard. We here introduce a physical-space formulation that captures unsteady advective and diffusive processes at all scales of the turbulent flow. The approach is a 3D construction based on the Linear Eddy Model (LEM), involving three orthogonally intersecting arrays of 1D LEM domains, and coupled so as to capture the 3D character of fluid trajectories. To illustrate the model performance of the 3D LEM-based formulation, here termed LEM3D, multi-stream mixing in a turbulent round jet is simulated using measured mean-flow properties as input. Comparison to scalar crosscorrelation coefficients and other measured properties of this mixing configuration indicate that the LEM3D approach, in conjunction with flow properties that are provided by steady-state models, is a useful representation of complex turbulent mixing processes that would otherwise be difficult to capture within a steady-state CFD framework.
\end{abstract}

Keywords Linear Eddy Model • Turbulent mixing • Subgrid scalar closure • Turbulent reactive flows

S. Sannan $(\bowtie) \cdot$ T. Weydahl

SINTEF Energy Research, 7465 Trondheim, Norway

e-mail: sigurd.sannan@sintef.no

A. R. Kerstein

Combustion Research Facility, Sandia National Laboratories, Livermore, CA 94551-0969, USA

Present Address:

A. R. Kerstein

72 Lomitas Road, Danville, CA 94526, USA 


\section{Introduction}

The Linear Eddy Model (LEM) developed by Kerstein [1, 2] was formulated to capture the principal mechanisms governing the turbulent mixing of diffusive scalars in a computationally affordable manner. The model can accommodate multiple species undergoing chemical reactions (see [3]), and explicitly distinguishes between turbulent advection, diffusive mixing, and chemical reaction at all scales of the turbulent flow. This feature is crucial in order to capture the dissimilar influences of these mechanisms on the mixing and combustion processes. The resolution of all relevant length scales in LEM is achieved by reducing the description of scalar fields to one spatial dimension.

Applications of 1D LEM have been limited to relatively simple flow configurations, such as flows that are homogeneous in at least one spatial direction [4]. For more complex flows, McMurtry et al. [5, 6] proposed to use LEM as a subgrid mixing and chemistry closure for Large Eddy Simulation (LES). In this modeling approach the unresolved length and time scales of LES are captured by individual LEM domains associated with each LES control volume, and these domains are coupled by the transfer of fluid elements from one domain to another as prescribed by LES-resolved mass fluxes. The complete LEM-LES model, with two-way coupling between LEM and LES, was later demonstrated for scalar mixing and non-premixed combustion [7], and has also been applied to premixed combustion [8, 9].

Despite increasing prospects for the use of LES in general to predict mixing and reaction in turbulent flows, it is of great practical interest to impart the desirable attributes of LES, namely unsteadiness and spatial resolution, to RANS methods to the extent that this is achievable. We here adapt the subgrid modeling strategy of LEM-LES for the purpose of representing unsteadiness and small-scale structure within a RANS framework. The use of LEM coupled to RANS has been proposed previously, but to date there has been limited demonstration and evaluation of this approach [10].

The present formulation, termed LEM3D, incorporates three orthogonally intersecting arrays of 1D LEM domains, with intersecting LEM domains coupled in a Lagrangian sense by non-diffusive fluid-cell displacements. LEM3D thus provides small-scale resolution in all three spatial directions of the turbulent flow field. The modeling approach has the potential of resolving all scales of turbulent reactive flows at a computationally affordable cost compared to a corresponding Direct Numerical Simulation (DNS). LEM3D is broadly analogous to LEM-LES in its physical treatment, but differs in its overall structure in ways that facilitate its future use as a flexible tool for mixing and chemical reaction simulation within 3D flow solutions.

To motivate and demonstrate the LEM3D approach, we here present its application to a challenging turbulent mixing configuration that has not previously been modeled. Tong and Warhaft [11], here denoted TW, performed an experiment that, in effect, measured the mixing of passive scalars emitted from various ring pairs embedded in a turbulent jet, including the cross-correlation of the passive scalars emitted from the respective ring sources. This configuration is analogous to earlier cross-correlation measurements of scalars emitted from line sources in homogeneous grid turbulence [12], with the additional complications of non-uniform mean flow and cylindrical vs. planar symmetry of the mean mixing. 
The paper is organized as follows. A description of the LEM3D model formulation, along with details of the numerical implementation, is presented in Section 2. The application of LEM3D to the TW rings experiment is then described in Section 3 , followed by a presentation of the results in Section 4. A discussion and assessment of the results is contained in Section 5. Further details of the LEM3D formulation are provided in the appendices at the end of the paper.

\section{LEM3D Model Formulation}

\subsection{The Linear Eddy Model}

The 1D LEM has been described in detail in previous work [2, 3], and only the main features are summarized here. The evolution of a scalar field $\phi(x, t)$ is governed by the equation

$$
\frac{\partial \phi}{\partial t}=\frac{\partial}{\partial x}\left(D_{M} \frac{\partial \phi}{\partial x}\right)+\mathcal{T}
$$

where $D_{M}$ is the molecular diffusivity, and $\mathcal{T}$ is a term representing turbulent advection (stirring). The model resolves all spatial and temporal scales, down to the smallest turbulence scales, and the above equation is solved exactly with Kolmogorov scale resolution. Additional resolution down to the Batchelor scale may be required for cases in which this is smaller than the Kolmorogov scale, although coarser resolution is acceptable if results are demonstrably insensitive to the coarsening.

The diffusive time advancement of Eq. 1 is punctuated by randomly occurring stirring events. These stochastic events, denoted triplet maps and represented by $\mathcal{T}$, emulate the effects of individual turbulent eddies on the scalar concentration fields. In Lagrangian terminology, the triplet maps rearrange fluid cells, represented by the computational cells of the discretized 1D domain, in such a manner that scalar length scales are reduced and local gradients are magnified. The triplet maps are sampled from an inertial-range size distribution and occur on randomly selected segments of the $1 \mathrm{D}$ domain.

In this paper we present the discretized versions of the parameters governing the stirring events, as implemented in LEM3D. The triplet map is here defined for segments of size $3 k$ cells, where $k$ is an integer. The stirring events introduce a random walk of the fluid cells of the $1 \mathrm{D}$ domain, with the associated diffusivity interpreted as the turbulent diff usivity $D_{T}$. This gives a relation between $D_{T}$ and the rate of stirring events, as derived in Appendix A. From Eq. 18 the event frequency parameter $\lambda$, with units (length $\times$ time $)^{-1}$, is given by

$$
\lambda=\frac{D_{T}}{2 d^{3}}\left(\sum_{k=k_{\min }}^{k_{\max }} k^{2}(k-1) f(k)\right)^{-1},
$$

where $d$ is the width of one fluid cell, and $f(k)$ is the probability function describing the eddy size distribution. The limits $k_{\min }$ and $k_{\max }$ in the above sum represent the smallest and largest eddy events, corresponding to the model analogues $L_{K}$ and $L$ of the Kolmogorov scale $\eta$ and the integral length scale $l$, respectively. Hence, the smallest eddies are given by $L_{K}=3 k_{\min } d$, while the largest eddies are given by 
$L=3 k_{\max } d$. The parameter $L$ is not precisely equal to the measured integral scale $l$, but is represented by $L=c l$, where $c$ is a parameter to be adjusted to give a best fit to scalar mixing properties. This issue has been thoroughly addressed by Kerstein $[2,3]$.

The specific form of $f(k)$ is determined by the requirement that the turbulent diffusivity obeys scaling laws in accordance with the Kolmogorov inertial-range cascade. The discretized version of the turbulent diff usivity, attributable to segments of size $3 \hat{k} d$ or less, is given by

$$
D_{T}(\hat{k})=2 \lambda d^{3} \sum_{k=k_{\min }}^{\hat{k}} k^{2}(k-1) f(k) .
$$

(In expressions where $D_{T}$ has no argument, $\hat{k}=k_{\max }$ is implied.) In the inertial range, corresponding to $k_{\min } \leq \hat{k} \leq k_{\max }$, the diff usivity induced by eddies of size $3 \hat{k} d$ or less scales as $\hat{k}^{p}$, where $p=4 / 3$ [13]. Thus, since to leading order $\sum_{k=k_{\min }}^{\hat{k}} k^{p-1} \propto \hat{k}^{p}$, this implies that the probability function behaves as $f(\hat{k}) \propto \hat{k}^{p-4}$. The normalized probability function in the inertial range is

$$
f(\hat{k})=\frac{\hat{k}^{p-4}}{\sum_{k=k_{\min }}^{k_{\max }} k^{p-4}} ; \quad k_{\min } \leq \hat{k} \leq k_{\max } .
$$

\subsection{Strategies for LEM coupling to 3D flow solutions}

McMurtry et al. [5] introduced a subgrid closure for mixing and chemistry based on LEM that has been used for LES closure in many subsequent studies (see e.g. [8,9]), and also for RANS closure in one study [10]. This closure introduces an LEM domain in each control volume of the 3D mesh. The LEM domain size is of the order of the resolution scale of the $3 \mathrm{D}$ mesh, and evolves a $1 \mathrm{D}$ profile of the thermochemical state that is deemed to be a representative sample of evolution within the control volume that contains it. Specializing to LES closure, the LES time-advancement cycle begins with an update of the coarse-grained LES flow state, consisting of velocity components and density. (All thermochemical information resides solely within the LEM domains.) Next, diffusive transport, chemistry, and triplet maps representing subgrid advection are implemented within each subgrid LEM domain for a time interval equal to the LES time step. Then the LEM domains communicate with each other by means of a 'splicing' procedure. The mass transfer across each LES mesh face during the LES time step is computed based on the LES-resolved velocity and density. The prescribed transfer is applied to the affected LEM domains by excising a piece of the domain that is upwind of the LES face and inserting it into the LEM domain that is downwind of the LES face. Details of this procedure, which are presented in the cited references, are not repeated here because the present approach is only indirectly related to splicing. A survey of LES/LEM applications to date is given by Menon and Kerstein [14]. Similar, if not greater, impact can be anticipated by coupling LEM3D to RANS or LES and eventually to ODTLES $[15,16]$. 
A relevant point for present purposes is that, in different implementations of the splicing strategy, LEM domains can have either periodic or Neumann boundary conditions. If the former, then spliced pieces can be excised and inserted at arbitrary locations or a type of first-in-first-out criterion can be applied. If the latter, then the LEM domain has an input side and an output side. A common characteristic of these implementations is that the LEM domains are Lagrangian objects that have no unique spatial location or orientation within the LES control volume that contains them.

In contrast, the formulation introduced here has a well-defined spatial structure. In fact, its structure is the same as in ODTLES [15, 16], a method for 3D flow advancement involving an array of coupled 1D domains, with the One-Dimensional Turbulence (ODT) stochastic flow simulation method [17] implemented on each. ODT involves a dynamic approach to triplet-map sampling that enables turbulentflow prediction, complementing the LEM simulation of scalar mixing within turbulence. One eventual goal of the present effort is to couple LEM3D to ODTLES. ODTLES will specify the triplet maps that are implemented concurrently on the ODT and LEM domains, where velocity evolves on ODT domains, thermochemistry evolves on LEM domains, and density is communicated from the latter to the former.

In ODTLES, 3D coarse-grained advection that couples the ODT domains is implemented using a finite-difference method that fluxes properties between domains using a coarse-grained spatial increment, such that the property gradients in the directions of the fluxes are underresolved. This and other steps in the advective advancement introduce numerical transport. The magnitude of this numerical transport is small enough so that resulting artifacts are minor. An analogous form of domain coupling in LEM3D would be undesirable because the resulting numerical transport could introduce more serious artifacts such as nonrepresentative or unrealizable chemical states. Such artifacts are common in conventional mixing models, but avoidance of these artifacts is the main justification for the extra complexity and cost of LEM3D. Like the splicing strategy, the LEM3D approach described here is expressly designed to avoid numerical transport and its consequences.

An advantage of LEM3D relative to the splicing strategy is that it avoids the imposition of Neumann boundary conditions on LEM within each 3D control volume, which is the currently preferred splicing formulation. A related advantage is that a triplet map need not be contained within one 3D control volume, nor need it be limited in size relative to the control volume. Also, the three-array substructure of LEM domains in LEM3D (which is explained in Section 2.3) has the potential of accommodating orientation-dependent processes such as buoyancy effects. An advantage of the splicing strategy is that it is readily implemented within an arbitrary structured or unstructured mesh.

A strategy that is presently being pursued to apply LEM3D to complex geometries is to implement it within a Cartesian subvolume within a mesh that is curvilinear outside that subvolume and uses either a conventional combustion submodel or the splicing method in the curvilinear region. This strategy will be well suited for situations in which the need for highly resolved mixing and chemistry is primarily in a region that is far from vessel walls.

As noted, LEM3D is complementary in structure to ODTLES, but can be applied more generally to LES or RANS closure. Here the focus is on demonstrating the capability of LEM3D to capture unsteadiness and small-scale resolution of scalar 
mixing using only mean-flow information analogous to the information that would be provided by a RANS solution. To avoid the complication of distinguishing LEM performance as a mixing model from RANS performance as a flow solver, the mean flow information in the present demonstration is based on experimental data rather than a RANS solution.

\subsection{The LEM3D geometry and numerical discretization}

In the current implementation of LEM3D, the global flow domain is assumed to be a cuboid of dimensions $X_{1}, X_{2}$, and $X_{3}$. The flow domain, denoted $X_{1} X_{2} X_{3}$, is discretized in two distinct but interdependent ways as follows. First, a Cartesian mesh is formed by cubic control volumes (3DCVs) of edge length $M$. This gives a discretization of $N_{1} N_{2} N_{3}$ 3DCVs, where $N_{1}=X_{1} / M, N_{2}=X_{2} / M$, and $N_{3}=X_{3} / M$. The second discretization is established by embedding three mutually orthogonal arrays of 1D LEM domains within the coarser 3D mesh. For instance, in the $x_{3}$ direction there is an array of $N_{1} N_{2}$ LEM domains $\Delta_{i, j}^{X_{3}}$ of length $X_{3}$. As illustrated in Fig. 1, each LEM domain is discretized into a finite number of equally sized LEM cells. The cells, denoted LEM wafers in the following, all have a volume $M^{2} d$, where $d$ is the wafer thickness and typically is of the order of the viscous scales. The fine-scale resolution is $d=M / n$, where $n$ is the number of LEM wafers in the intersection of

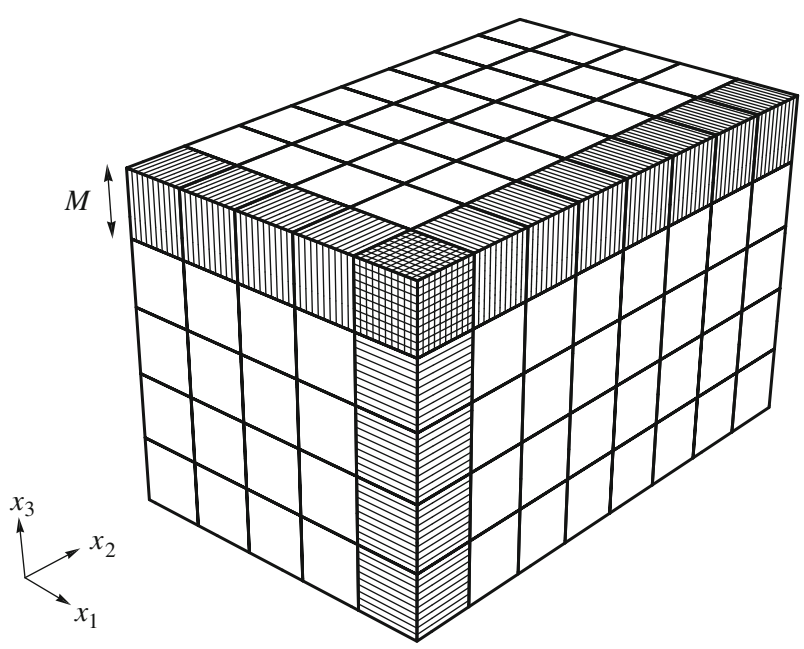

Fig. 1 A subregion of the flow domain $X_{1} X_{2} X_{3}$ illustrating the discretization into a Cartesian mesh and a superimposed fine-scale resolution by orthogonal arrays of LEM domains in each spatial direction $x_{k}(k=1,2,3)$. The cubic control volumes (3DCVs) correspond to intersections of sets of three orthogonal LEM domains and their edge lengths are denoted $M$. In this illustration the number of LEM wafers per 3DCV in each coordinate direction is $n=10$. Note that the 3DCV in the top-front corner suggests a partition into $n^{3}$ subvolumes. However, in LEM3D, each LEM domain that intersects a $3 \mathrm{DCV}$ partitions the $3 \mathrm{DCV}$ in the corresponding direction and is part of a different flow solution than those associated with the other intersecting LEM domains of the 3DCV. Hence, three separate flow solutions are obtained and the number of LEM wafers per $3 \mathrm{DCV}$ is $3 n$ vs. $n^{3}$ for a partition corresponding to a notional DNS of the flow 
an LEM domain with a 3DCV. The array of LEM domains $\Delta_{i, j}^{X_{3}}$ fills the entire cuboid $X_{1} X_{2} X_{3}$ and resolves turbulent stirring and molecular mixing in the $x_{3}$-direction. The arrays of orthogonally oriented LEM domains provide equal fine-scale resolution in the orthogonal directions. Thus, in the $x_{1}$-direction there is an array of $N_{2} N_{3}$ domains $\Delta_{j, k}^{X_{1}}$, each of length $X_{1}$, while in the $x_{2}$-direction there is an array of $N_{1} N_{3}$ domains $\Delta_{k, i}^{X_{2}}$, each of length $X_{2}$. With equal resolution in all three spatial directions, the total number of LEM wafers in the domains $\Delta_{j, k}^{X_{1}}, \Delta_{k, i}^{X_{2}}$, and $\Delta_{i, j}^{X_{3}}$ is $n N_{1}, n N_{2}$, and $n N_{3}$, respectively.

In this configuration, each 3DCV of the Cartesian mesh is intersected by three orthogonal LEM domains. The 3DCVs are thus resolved in total by $3 n$ LEM wafers, with $n$ wafers for each coordinate direction. The total number of LEM wafers resolving the flow domain $X_{1} X_{2} X_{3}$ is $3 n N_{1} N_{2} N_{3}$. In comparison, a corresponding DNS of the domain would require $n^{3} N_{1} N_{2} N_{3}$ grid cells. The computational cost saving achieved with LEM3D compared to a DNS is therefore of order $n^{2}$.

The LEM3D model inputs are the parameters $D_{M}, L, L_{K}$, and $p$, along with the turbulent diffusivity field $D_{T}$ and the mean velocity field $\boldsymbol{U}$. The mean velocity governs the advective transport of the scalars, and both the diffusivity $D_{T}$ and the velocity $\boldsymbol{U}$ generally vary in the spatial directions. The turbulence and velocity field inputs to LEM3D are resolved at the coarser length scale $M$ only, with the values of $D_{T}$ fed to the centers of the 3DCVs and the face-normal components of $\boldsymbol{U}$ provided to the faces of the 3DCVs. As explained in Appendix B, the advective transport due to the velocity $\boldsymbol{U}$ involves a transfer of LEM wafers across the interfaces between neighboring 3DCVs. This transfer of wafers provides an advective coupling between neighboring LEM domains. An additional auxiliary coupling of the LEM domains is implemented by stochastic rotations of the 3DCVs, as described in Appendix C.

The cuboid $X_{1} X_{2} X_{3}$ in which LEM refinement is implemented may in general be a subdomain of a larger flow domain where a RANS or an LES flow solver is applied. By this approach, the extended CFD domain can be a complex geometry with arbitrary meshing and conventional combustion closure in the region outside the LEM3D cuboidal subdomain, and with interpolation applied as needed to transfer information between the arbitrary mesh and the LEM array structure. The appropriate meshing features of the extended flow domain, e.g., a curvilinear or an unstructured grid, are available in commercial CFD software. This approach, where the LEM3D substructure is coupled to a global flow solver, will be well suited to applications for which there is a need for high resolution of important chemistrytransport details in a region far from the combustion chamber walls. In the current LEM3D stand-alone application to a turbulent jet, experimental data expressed in 2D axi-symmetric coordinates are converted to match the 3D Cartesian mesh.

A two-way coupling between LEM3D and RANS is presently being developed. The coupling is based on a variable-density formulation that allows thermal expansions and involves an iterative process in which updated information obtained from the steady-state solution of either tool is fed back into the other as input. Thus, a RANS solution provides LEM3D with updated velocity and turbulence fields, while a LEM3D solution provides RANS with an updated mean density field obtained by time averaging after LEM3D relaxation to statistically steady time advancement. The iterative process of the variable-density coupling is continued until convergence is achieved for all relevant physical quantities. 


\subsection{The transport equations}

In the present formulation we focus on a zero-Mach-number incompressible turbulent flow. The evolution of a non-reactive scalar $\phi_{l, m}^{(k)}$ on an LEM domain $\Delta_{l, m}^{X_{k}}$ oriented in the $x_{k}$-direction is governed by the equation

$$
\frac{\partial \phi_{l, m}^{(k)}}{\partial t}=\frac{\partial}{\partial x_{k}}\left(D_{M} \frac{\partial \phi_{l, m}^{(k)}}{\partial x_{k}}\right)+\mathcal{T}_{l, m}^{(k)}+\mathcal{A} \mathcal{D} \mathcal{V}_{3 \mathrm{D}},
$$

where the molecular diff usive transport, controlled by $D_{M}$, is supplemented by triplet maps $\mathcal{T}_{l, m}^{(k)}$ and a term $\mathcal{A} \mathcal{D} \mathcal{V}_{3 \mathrm{D}}$ denoting advective coupling of intersecting LEM domains. Note that repeated indices $k$ in the above equation do not imply summation. The rate of occurrence of triplet maps $\mathcal{T}_{l, m}^{(k)}$ at a given location is determined by the turbulent diffusivity $D_{T}$ at that location, so the eddy event frequency $\lambda$, as given by the expression (2), varies with $x_{k}$. Triplet-map sizes are sampled from the probability distribution (4) which represents turbulent inertial-range phenomenology.

The molecular-diffusive and turbulent transport of Eq. 5 is time advanced for each LEM domain $\Delta_{l, m}^{X_{k}}$ of the three orthogonal arrays of domains. The time advancement involves an LEM sub-cycling within every time step $\Delta t$ of the advection advancements, represented by the term $\mathcal{A D} \mathcal{V}_{3 \mathrm{D}}$. The advection term $\mathcal{A D} \mathcal{V}_{3 \mathrm{D}}$ consists of two operations given by

$$
\mathcal{A D} \mathcal{V}_{3 \mathrm{D}}=\mathcal{A D} \mathcal{V}+\mathcal{A C}
$$

where $\mathcal{A D} \mathcal{V}$ accounts for the Lagrangian advection of LEM wafers based on the mean velocity field $\boldsymbol{U}$, and $\mathcal{A C}$ represents an auxiliary coupling of orthogonally intersecting LEM domains. The auxiliary coupling, implemented by stochastic rotations of the 3DCVs, ensures that physical processes are consistently represented in all spatial directions. The operations $\mathcal{A D} \mathcal{V}$ and $\mathcal{A C}$ are discussed in more detail in Appendices B and C.

The evolution of the scalar fields $\phi_{l, m}^{(k)}(k=1,2,3)$ through the operator-splitting scheme of Eq. 5 gives three distinct solutions for the generic scalar field $\phi$, one per domain orientation. Thus, three correlated copies of the scalar statistics are obtained. Although the scalar fields $\phi_{l, m}^{(k)}$ are closely coupled, they can be treated as distinct realizations for data reduction purposes.

\section{Application of LEM3D to Mixing from Localized Sources in a Turbulent Round Jet}

\subsection{Motivation}

Three-stream mixing is not readily modeled using mixture-fraction-based methods because two parameters rather than one are needed to specify the composition of a fluid element. For this reason, three-stream mixing is a conceptually as well as practically important test case for mixing models. A foundational three-stream mixing study involving the mixing of two distinct passive scalars emitted by line sources in grid turbulence was performed by Warhaft [12]. (The experiment actually involved a single scalar property, heat, but results of one-source and two-source measurements were combined so that the outcome was equivalent to the mixing 
of distinct scalars emitted from the respective lines.) The geometrical simplicity of this configuration allowed for the simulation of measured mixing properties using a single LEM domain [3]. Recently, Viswanathan and Pope [18] demonstrated that this configuration can also be modeled using state-space closure methods.

Tong and Warhaft [11] used the same heated-wire technique as in the line-sources experiment to emulate the mixing of distinct scalars emitted by each of two rings embedded in a turbulent jet. Neither the flow nor the scalar-source geometry can be captured on a single LEM domain. LEM3D is designed specifically to capture more general mixing geometries such as the TW configuration. Accordingly, the TW rings experiment, which has not previously been modeled, is chosen as an illustrative application.

It should be noted that this application is more challenging in some respects than the applications for which the present formulation is ultimately intended. The reason is that the 3DCVs must be small enough to resolve the individual rings, and that for two of the TW configurations the ring pairs are as close as $2.5 \mathrm{~mm}$ apart. Thus, the dimensions of the rings impose a constraint, and a relatively fine 3DCV mesh with $M=2 \mathrm{~mm}$ is applied for these configurations. As explained in Appendix C, it is desirable in LEM3D that the 3DCVs are as large as possible in order to minimize the frequency of domain-coupling operations, thereby mitigating artifacts associated with these couplings.

Although LEM3D is designed to be complementary to RANS, for this application the needed mean flow properties are obtained from measurements rather than from a RANS simulation. This approach is adopted because the goal is to illustrate the performance of LEM3D without the added complication of discrepancies resulting from imperfect prediction of mean flow properties by RANS models.

\subsection{LEM3D application to the TW experiment}

TW used heated fine wire rings as passive-scalar sources in order to study dispersion and mixing in a turbulent jet. The scalars are the temperature fluctuations produced by the rings, placed axisymmetrically and in the self-similar region of the turbulent flow. The diameters of the rings were typically of the order of the jet diameter $D_{j}$, and the rings were located at a distance $x_{\text {ring }}=9 D_{j}$ downstream of the jet exit. A jet diameter of $D_{j}=30 \mathrm{~mm}$ was used in the heated rings study, and the jet exit velocity was $U_{j}=9 \mathrm{~m} / \mathrm{s}$.

In the application presented here, the mean flow properties used as model input to LEM3D were generated on the basis of jet velocity and turbulence measurements. Thus, the mean centerline velocity is estimated to decay as

$$
U_{c}=5.98 U_{j}\left[\left(x-x_{0}\right) / D_{j}\right]^{-1},
$$

based on data shown in Fig. 3a of TW, and where $x_{0}=-0.61 D_{j}$ is the virtual origin of the jet. Measurements made by Panchapakesan and Lamley [19] indicate that the axial mean velocity profile across the jet can be well approximated with a Gaussian distribution for a suitable half-width. We here use the jet half-width $l_{1 / 2}=28 \mathrm{~mm}$ measured at $x_{\text {ring }}$ (see TW) to specify the Gaussian profile of the axial velocity $U\left(x_{\text {ring }}, r\right)$. From this, and Eq. 7, the self-similar profile of the axial velocity $U(x, r)$ of the jet was generated in cylindrical coordinates using the software 
program Mathematica [20]. The self-similar radial velocity profile $V(x, r)$ of the jet was obtained using the continuity equation

$$
\frac{1}{r} \partial_{r}(r V)+\partial_{x} U=0
$$

where axisymmetry requires that $V(x, 0)=0$. Similarly, the turbulent diffusivity $D_{T}$ is obtained on the basis of the Reynolds stress $\langle u v\rangle$ shown in Figure 12 of [19]. The Reynolds stress is generalized to a self-similar function in the domain with the appropriate fall-off in the streamwise direction in order to ensure conservation of momentum flux. In the boundary-layer approximation this gives the turbulent diffusivity

$$
D_{T}(x, r)=-\frac{\langle u v\rangle}{P r_{T} \partial_{r} U},
$$

where $\operatorname{Pr}_{T}$ is the turbulent Prandtl number and terms containing other Reynoldsstress components are neglected, both because the magnitudes of these terms are small and because the empirical basis for their evaluation is limited. The turbulent Prandtl number is an empirical quantity, which we here set to $\operatorname{Pr}_{T}=1$ because there is no empirical basis for associating a non-unity value with a localized source in the interior of a turbulent jet.

In the absence of a physical measurement of the integral scale, the model integral scale $L$ is chosen as twice the half-width of the jet measured at $x_{\text {ring }}$ by TW. Thus, $L=56 \mathrm{~mm}$, and is here assumed to be a constant throughout the computational domain. This choice provides the best agreement between the model and measurements among several $L$ values that were tested. The kinematic viscosity is taken as $v=1.5 \times 10^{-5} \mathrm{~m}^{2} / \mathrm{s}$, and the Schmidt number is $S c=0.75$. Hence, the molecular diffusivity is

$$
D_{M}=\frac{v}{S c}=2.0 \times 10^{-5} \mathrm{~m}^{2} / \mathrm{s} .
$$

For the present application, the flow domain $X_{1} X_{2} X_{3}$ is discretized by $31 \times 31 \times$ 50 3DCVs, each of size $M=8 \mathrm{~mm}$, as the baseline case. The coarse-scale mesh, denoted M8, was chosen because it is just fine enough to give a distinct representation of the TW ring pairs of diameters 10, $30 \mathrm{~mm}$ and 20, $40 \mathrm{~mm}$, respectively. For the 10, $15 \mathrm{~mm}$ and 35, $40 \mathrm{~mm}$ ring pairs, however, the M8 mesh is too coarse to represent the rings as distinct sources. Hence, in order to resolve the small spacing between the rings of these configurations, a finer mesh with 3DCVs of size $M=2 \mathrm{~mm}$, is applied. The fine mesh, here denoted M2, is discretized by $121 \times 121 \times 2003 \mathrm{DCVs}$, and is applied in additional simulations that involve all the ring pairs. The physical volume of the domain $X_{1} X_{2} X_{3}$ is roughly the same whether the M8 or the M2 mesh is applied. It is worth noting that the ring sources in either case are crudely represented by the coarse-scale resolution.

The fine-scale LEM resolution is chosen as $n=40$ and $n=10$, respectively, for the M8 and the M2 meshes. This yields the same wafer thickness $d=0.2 \mathrm{~mm}$ for both meshes. With $L=56 \mathrm{~mm}$, the largest eddy events in the simulations involve 279 LEM wafers, corresponding to $k_{\max }=93$ in Eq. 2, while the smallest eddy events involve $12 \mathrm{LEM}$ wafers, corresponding to $k_{\min }=4$ in Eq. 2 . The minimum value $k_{\text {min }}=4$ is chosen because triplet maps corresponding to the smallest possible value $k_{\min }=2$ (and to $k_{\min }=3$ to a lesser degree) produce unphysical ragged profiles in 
some cases. The fine-scale resolution implies that the resolved length scale in the application is somewhat larger than the Kolmogorov scale. In TW the Kolmogorov scale for the turbulent jet is estimated to $\eta=0.08 \mathrm{~mm}$, while the smallest triplet maps in the simulation are of size $L_{k}=2.4 \mathrm{~mm}$, hence $L_{k}=30 \eta$. On the other hand, the wafer thickness $d$ is of the order of the Kolmogorov scale and given by $d=2.5 \eta$. The important consideration here is that results are not very sensitive to the size of the smallest LEM eddy. In LEM, the mixing properties considered here rapidly approach their high-Reynolds-number limiting behaviors for $L_{k}<<L$. In turbulent flows the convergence is slower because the onset of inertial-range behavior is gradual as $R e \sim\left(L / L_{k}\right)^{4 / 3}$ increases. This slow onset could be replicated in LEM using empirically based finite- $R e$ modifications of the assumed inertial-range transport scaling, but for present purposes this additional nuance is not needed.

The global advancement time step is

$$
\Delta t=\frac{\mathrm{CFL} M}{V_{\max }},
$$

where CFL is the dimensionless Courant-Friedrichs-Lewy number, and $V_{\max }$ is the maximum velocity component (by absolute value) associated with any one of the 3DCV side faces of the computational domain. In the simulations shown here, $\mathrm{CFL}=0.1$ and $V_{\max }=20.9 \mathrm{~m} / \mathrm{s}$. The magnitude of $V_{\max }$ incorporates the fact that the physical velocity components $U_{k}$ (and the turbulent diffusivity $D_{T}$ ) are multiplied by a factor 3 in LEM3D, i.e., the implemented velocity components are given by $V_{k}=3 U_{k}$. This is done to compensate for the fact that, due to the coupling between the differently oriented LEM domains, the LEM wafers on average spend only one third of the time in any one of the coordinate directions. It follows from Eq. 11 that the time step for the baseline cases with the M8 mesh is $\Delta t=3.83 \times 10^{-5} \mathrm{~s}$. For the results shown here the simulation runs for the M8 cases were over 200000 time-advancement cycles, with data collection every 50 cycles starting at 30000 cycles so that initial transient relaxation is excluded. The time step for the M2 mesh is $\Delta t=9.56 \times 10^{-6} \mathrm{~s}$, and the runs are over 250000 cycles, with data collection starting at 60000 cycles with a period of 200 cycles.

\section{Results}

\subsection{The scalar field emitted from a single ring}

In the heated-rings experiment, TW studied both dispersion and mixing of temperature fluctuations from rings of different diameters placed in a turbulent jet of diameter $D_{j}=30 \mathrm{~mm}$ and exit velocity $U_{j}=9 \mathrm{~m} / \mathrm{s}$. Single rings were used to study dispersion, while pairs of rings were used to study the mixing of two scalar fields introduced independently into the jet.

We first consider LEM3D simulation results for single rings with the use of the baseline M8 mesh. Figure 2 shows the evolution of the mean temperature $T_{c}$ along the jet centerline for rings of diameters 10, 20, 30, and $40 \mathrm{~mm}$ embedded in the jet. Following TW, the constant $C_{r}$ is chosen for each ring to effectively match the heat flux in each case. In agreement with the measurements, the simulations show that the quantity $T_{c} x$ approaches a horizontal line at the normalized downstream 
Fig. 2 The evolution of the centerline mean temperature for the 10, 20,30, and $40 \mathrm{~mm}$ rings. The rings were placed at $x_{\text {ring }} / D_{j}=9$ in a turbulent jet of $D_{j}=30 \mathrm{~mm}$ and exit velocity $U_{j}=9 \mathrm{~m} / \mathrm{s}$. The curves show the simulated profiles, while the measurements are given as follows: $\bigcirc, 10 \mathrm{~mm}$ ring; $\square, 20$ $\mathrm{mm}$ ring; $\diamond, 30 \mathrm{~mm}$ ring; $\triangle, 40$ $\mathrm{mm}$ ring

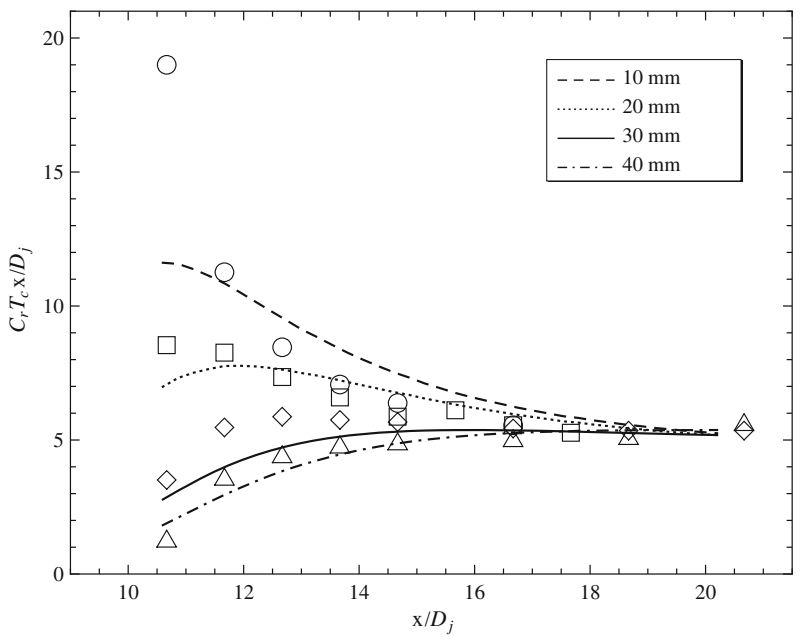

distance $x / D_{j} \approx 20$. The simulations also show that the centerline temperature is high immediately downstream of the small 10 and $20 \mathrm{~mm}$ rings, while the initial values are low for the larger 30 and $40 \mathrm{~mm}$ rings. This is due to the fact that the peak mean temperatures for the large-diameter rings occur off the jet centerline for $x / D_{j} \leq 13$ (see Fig. 4).

The centerline scalar fluctuation intensity $\left\langle\theta^{2}\right\rangle^{1 / 2} / T_{c}$ is shown in Fig. 3, where $\theta$ is the temperature fluctuation about $T_{c}$. We observe that the fluctuation intensity is large close to the rings but decreases rapidly in the downstream direction. At $x / D_{j} \approx 20$, the fluctuation intensity approaches a common value for all the different rings, reflecting the approach to self-similarity that is also seen in the mean temperature profiles in this vicinity (see Fig. 2). In agreement with the experimental results, it takes a longer time for the smaller rings to reach the asymptotic value than for the larger rings.

One obvious observation from Fig. 3 is that the LEM3D fluctuation intensity is smaller than the measured fluctuations. This may be a consequence of the numerical implementation features that cause LEM3D advection scales to be generally smaller than in the corresponding turbulent flow. In particular, it is desirable for the 3DCV scale $M$ to be of the order of the LEM integral scale $L$ so that $3 \mathrm{D}$ advection advancement corresponds to mean motion and LEM advancement captures turbulence effects. Here, $M$ must be substantially smaller than this in order to resolve the individual rings, resulting in reduction of the effective scales of motion so that turbulent dissipation of scalar fluctuations is enhanced relative to turbulent production of these fluctuations. The occurrence of triplet maps of size as large as $L$ suggests that the large-scale motions are retained, but implementation of a size$L$ map, for instance, on one LEM domain introduces scalar differences relative neighboring domains, hence scale- $M$ fluctuations. Thus, for $M \ll L$, a size- $L$ map does not have a purely large-scale character. One remedy would be to implement fully $3 \mathrm{D}$ maps for scales $>M$, a possibility that has been noted and discussed by Goldin et al. [10] (though large $M$ would still be desirable for the reasons discussed 
Fig. 3 The evolution of the centerline scalar fluctuation intensity $\left\langle\theta^{2}\right\rangle^{1 / 2} / T_{c}$ for the 10 , 20,30 , and $40 \mathrm{~mm}$ rings placed at $x_{\text {ring }} / D_{j}=9$ in the turbulent jet. The curves show the simulated profiles, while the measurements are given as follows: $\bigcirc, 10 \mathrm{~mm}$ ring; $\square, 20$ $\mathrm{mm}$ ring; $\diamond, 30 \mathrm{~mm}$ ring; $\triangle, 40$ $\mathrm{mm}$ ring

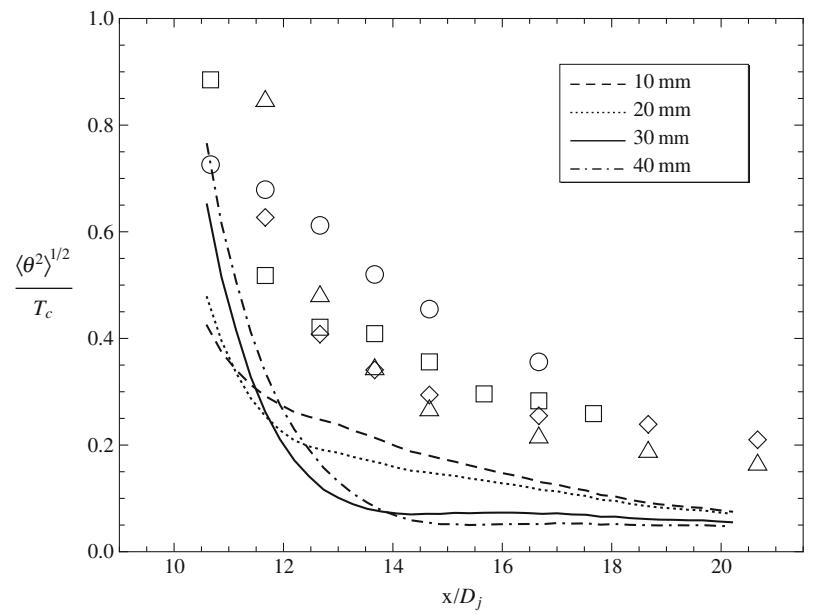

in Appendix C). This merits further investigation, but as an initial step we focus here on evaluation of LEM3D without this additional feature.

Despite the low fluctuations, the curve crossings between the individual rings shown in Fig. 3 agree well with the location of the corresponding crossings obtained in the measurements. For instance, the simulated centerline fluctuation intensity of the $40 \mathrm{~mm}$ ring crosses the curves of the 10,20 , and $30 \mathrm{~mm}$ rings at $x / D_{j}=12.0,12.5$, and 13.9, respectively, while the corresponding curve crossings in the measurements occur at $x / D_{j}=12.2,13.2$, and 13.9.

In Fig. 4 are shown the transverse mean temperature profiles at various locations downstream of the $40 \mathrm{~mm}$ ring. We note that the peak at $x / D_{j}=11$ is at a higher level than what is measured in the TW experiment. The near-field discrepancy is a known model artifact of LEM resulting from the instantaneous nature of the eddy events [3]. Farther downstream, in the far-field range $x / D_{j} \geq 15$, we observe that the simulated profiles agree well with the nearly self-similar profiles measured by TW. The transverse r.m.s. profiles $\left\langle\theta^{2}\right\rangle^{1 / 2} / T_{c}$ at various locations $x / D_{j}$ downstream of the $40 \mathrm{~mm}$ ring are shown in Fig. 5. We notice again that the simulated r.m.s. values are generally lower than the experimentally measured values. Despite this, as shown in the following, LEM3D is able to capture well the cross-correlations between the temperature fluctuations of the TW ring sources. This reflects the fact that LEM3D does not involve a mixing parametrization, which typically relies on accurate prediction of scalar r.m.s. fluctuations.

\subsection{Mixing of scalars emitted from pairs of rings}

A central part of the TW rings experiment is the determination of the crosscorrelation coefficient $\rho$ describing the mixing of passive scalars in the turbulent jet. To this end, TW used ring pairs to indirectly infer the mixing statistics of two independent scalars (thermal fields) in the jet. The inference method [12] enabled the determination of mixing statistics of distinct passive scalars emitted by the respective rings by mathematical manipulation of three sets of temperature measurements in which the inner ring, the outer ring, or both rings are heated. In the corresponding 
Fig. 4 Transverse mean temperature profiles at various locations $x$ downstream of the $40 \mathrm{~mm}$ ring placed at $x_{\text {ring }} / D_{j}=9$ in the turbulent jet. The curves show the simulated profiles, while the measurements are given for the various downstream distances as follows:

$\bigcirc, x / D_{j}=11 ; \square, x / D_{j}=13$;

$\triangle, x / D_{j}=15 ; \diamond, x / D_{j}=17$

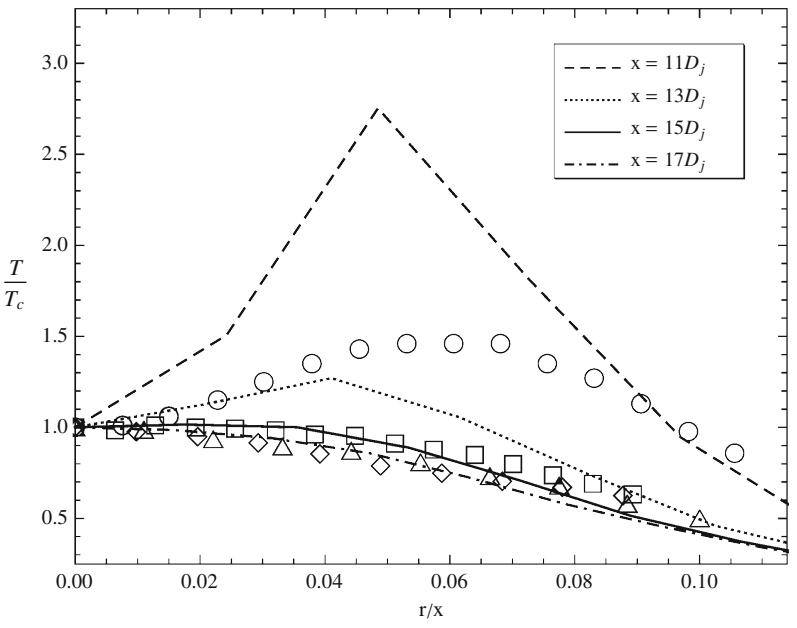

LEM3D simulations, it is convenient to introduce distinct scalar fields $A$ and $B$ associated with the respective rings rather than using the inference method.

The diameters of the TW ring pairs were $10,15 \mathrm{~mm}, 10,30 \mathrm{~mm}, 20,40 \mathrm{~mm}$, and $35,40 \mathrm{~mm}$, respectively, with the implication that the difference between ring diameters is small for two of the cases and large for the other two cases. In the simulation runs, a relatively fine $3 \mathrm{DCV}$ mesh with $M=2 \mathrm{~mm}$ is applied in order to resolve the closely spaced $10,15 \mathrm{~mm}$ and $35,40 \mathrm{~mm}$ pairs as distinct sources. The physical dimensions of the rings thereby impose a constraint on the Cartesian mesh in this application, effectively restricting the possibility of larger 3DCVs, which would be beneficial from a modeling viewpoint (see Appendix $\mathrm{C}$ for a discussion). Another implication is that the simulations for the rings mixing configuration are computationally more costly than otherwise needed.

Fig. 5 Transverse r.m.s. temperature profiles at various locations $x$ downstream of the $40 \mathrm{~mm}$ ring placed at $x_{\text {ring }} / D_{j}=9$ in the turbulent jet. The curves show the simulated profiles, while the measurements are given for the downstream distances as follows: $\bigcirc, x / D_{j}=11$;

$\square, x / D_{j}=13 ; \triangle, x / D_{j}=15$; $\diamond, x / D_{j}=17$




The correlation coefficient of scalar fields $A$ and $B$ is defined by

$$
\rho \equiv \frac{\left\langle\theta_{A} \theta_{B}\right\rangle}{\left\langle\theta_{A}^{2}\right\rangle^{1 / 2}\left\langle\theta_{B}^{2}\right\rangle^{1 / 2}},
$$

where $\theta_{A}$ and $\theta_{B}$ are the fluctuations about the means of the respective scalars. Figure 6 shows the cross-correlation coefficient of the scalar fluctuations along the jet centerline for the 20,40 $\mathrm{mm}$ and 10,30 $\mathrm{mm}$ ring pairs embedded in the turbulent jet. The baseline M8 mesh, which is too coarse to give a distinct representation of the $10,15 \mathrm{~mm}$ and 35, $40 \mathrm{~mm}$ ring pairs, is applied in these simulations. We note that the simulated correlation coefficients compare reasonably well with the experimentally obtained profiles for the coarse-spaced ring pairs. A common feature is that for both ring pairs the cross-correlation $\rho$ dips to negative values before it turns positive a little farther downstream. Negative cross-correlation signifies that the concentrations of scalars emitted by the respective rings are anti-correlated, i.e., a large concentration of the scalar from the source $A$ is associated with a small concentration of the scalar from the source $B$, and vice versa. This occurs where the micro-mixing (molecular mixing) of the $A$ and $B$ scalars is less thorough than their macro-mixing, which is related to the mechanisms of production and dissipation of scalar fluctuations but also is sensitive to the mean flow and the source configuration. Figure 6 shows that there is a good agreement between simulated and measured values for the magnitude of the maximum anti-correlation. In addition, the downstream location of the maximum anti-correlation is fairly well predicted by the simulations, with a deviation of a distance of about $D_{j} / 2$ in both cases. The TW measurements show that the correlation coefficient $\rho$ equals zero at the downstream distance $x / D_{j}=14$ for the 20, $40 \mathrm{~mm}$ ring pair, and at $x / D_{j}=13$ for the $10,30 \mathrm{~mm}$ ring pair. When $\rho=0$ there is no correlation between the scalar fluctuations of the two ring sources. We observe that the baseline M8 mesh simulations give fairly precise predictions of the locations of vanishing $\rho$ for both the coarse-spaced ring pairs. The main discrepancy between the simulated and measured centerline $\rho$ values shown in Fig. 6 is that the simulations overpredict the cross-correlations somewhat in the downstream range

Fig. 6 The evolution of the centerline cross-correlation coefficient $\rho$ as a function of $x / D_{j}$ for pairs of rings placed at $x_{\text {ring }} / D_{j}=9$ in the turbulent jet. The curves show the simulated results for the $20,40 \mathrm{~mm}$ and the $10,30 \mathrm{~mm}$ ring pairs with the M8 mesh, while the corresponding measurements are given as: 口, 20, $40 \mathrm{~mm} ; \square, 10,30 \mathrm{~mm}$




immediately beyond the point of vanishing $\rho$. The simulated curves are steeper than what is indicated by the measurements, i.e., higher $\rho$-values are attained faster. This reflects the excess of scale- $M$ vs. larger-scale mixing in this LEM3D configuration that was noted earlier. This imbalance accentuates the mixing of the $A$ and $B$ scalars, which are initially separated by a distance of order $M$, with each other relative to the mixing of either with the surrounding fluid. In the far-field downstream range $x / D_{j} \geq 18$, both the simulated and measured values of $\rho$ approach unity, reflecting the eventual merging of the initially separate $A$ and $B$ scalars.

Figure 7 shows the cross-correlation $\rho$ along the jet centerline for all the TW ring pairs in the turbulent jet. The M2 mesh is applied in these simulations since it is fine enough to represent also the finer-spaced $10,15 \mathrm{~mm}$ and $35,40 \mathrm{~mm}$ ring pairs as distinct sources. Figure 7 shows that the centerline evolution of $\rho$ for the $20,40 \mathrm{~mm}$ and 10,30 mm ring pairs is similar to that obtained for the M8 mesh. One difference, however, is that $\rho$ dips to higher negative values with the use of the M2 mesh. Another difference between the M2 and M8 results is that the M2 simulations do not equally well predict the downstream distances for which $\rho=0$ for the coarse-spaced TW rings. These deviations seem a bit arbitrary, though, since for the $20,40 \mathrm{~mm}$ ring pair the location of vanishing $\rho$ is slightly downstream of the experimentally obtained location, while for the $10,30 \mathrm{~mm}$ ring pair it is slightly upstream of the measured location. A more important difference is that the transition range from regions of high anti-correlation to regions of high correlation of the scalar fluctuations is shorter with the use of the M2 mesh. Also, since higher values of maximum anti-correlation are attained, the simulated curves are relatively steep in the transition range, reflecting the excessive degree of small-scale mixing for small $M$. Accordingly, case M8, with larger though still small $M$, gives a more realistic representation of the measured downstream mixing for the coarse-spaced rings in the turbulent jet (Fig. 6). Nevertheless, we see from Fig. 7 that the M2 simulation gives a fairly precise prediction of the downstream mixing process for the $10,15 \mathrm{~mm}$ ring pair. Note that values of $\rho>1$ shown at $x / D_{j}=15$ and 16 in the corresponding Fig. 9 of TW have been omitted here, but this is obviously of no practical importance

Fig. 7 The evolution of the centerline cross-correlation coefficient $\rho$ as a function of $x / D_{j}$ for pairs of rings placed at $x_{\text {ring }} / D_{j}=9$ in the turbulent jet. The curves show the simulated results for the ring pairs with the M2 mesh, while the measurements are given as follows: $\square, 20,40 \mathrm{~mm}$; $\square, 10,30 \mathrm{~mm}$; $, 10,15 \mathrm{~mm}$;

○, $35,40 \mathrm{~mm}$




for the comparison. The LEM3D jet centerline cross-correlation for the $35,40 \mathrm{~mm}$ ring pair is also in good agreement with the measurements, with a small overprediction in the near field and a dip below the measured results farther downstream.

The nature of the dip is of interest, and additional M2 simulations using 25, 40 $\mathrm{mm}$ and $30,40 \mathrm{~mm}$ ring pairs were performed to gain further understanding of its occurrence. Figure 8 shows the centerline cross-correlation for the series of ring pairs of diameters 20, $40 \mathrm{~mm}, 25,40 \mathrm{~mm}, 30,40 \mathrm{~mm}$, and 35, $40 \mathrm{~mm}$. From the centerline profiles it is evident that the dip in $\rho$ for the $35,40 \mathrm{~mm}$ pair is of the same origin as the dip to negative $\rho$-values for the $20,40 \mathrm{~mm}$ pair (and also for the $10,30 \mathrm{~mm}$ pair shown in Fig. 6). Namely, it reflects the arrival at the centerline of $A$ and $B$ scalars that are not molecularly well mixed, where this arrival occurs increasingly far downstream as the inner ring diameter increases due to the larger radial distance that the scalar from that ring must traverse. Later arrival implies more residence time during which micro-mixing can occur, implying a shallower dip and possibly the disappearance of the dip. (Decreasing separation of the rings promotes micro-mixing of the scalars, which accentuates the trend.) The measurements indicate such disappearance for the $35 \mathrm{~mm}$ inner ring, but the simulated ring-pair sequence suggests that measurements would detect dips for some range of inner-ring diameters between 20 and $35 \mathrm{~mm}$. Thus it is seen that the dip for the $35,40 \mathrm{~mm}$ pair is part of a reasonable trend though there is some quantitative disagreement with the measurements.

In Figs. 9 and 10 are shown transverse profiles of $\rho$ for the $10,15 \mathrm{~mm}$ and the $35,40 \mathrm{~mm}$ ring pairs, respectively. The simulation results are obtained by the use of the M2 mesh since the M8 mesh is too coarse to distinguish the fine-spaced rings as distinct sources. TW does not provide measured transverse profiles of $\rho$ for the other two ring pairs. We note that there is good overall agreement between the simulated and measured transverse profiles of the cross-correlation. In particular, the simulations capture essentially all the qualitative features of the measured profiles. TW discusses some of these features, notably those related to the transverse variation of mean shear in the jet, and some reflect the same mixing processes that affect the centerline profile features discussed above. Therefore the discussion here is brief.

Fig. 8 The evolution of the centerline cross-correlation coefficient $\rho$ as a function of $x / D_{j}$ for pairs of rings placed at $x_{\text {ring }} / D_{j}=9$ in the turbulent jet. The curves show the simulated results of ring pairs for which the diameter of the inner ring varies from 20 to $35 \mathrm{~mm}$, while the diameter of the outer ring is fixed at 40 $\mathrm{mm}$. Measurements are given only for two of the ring configurations with diameters as follows: $\mathbf{\square}, 20,40 \mathrm{~mm}$;

○, $35,40 \mathrm{~mm}$

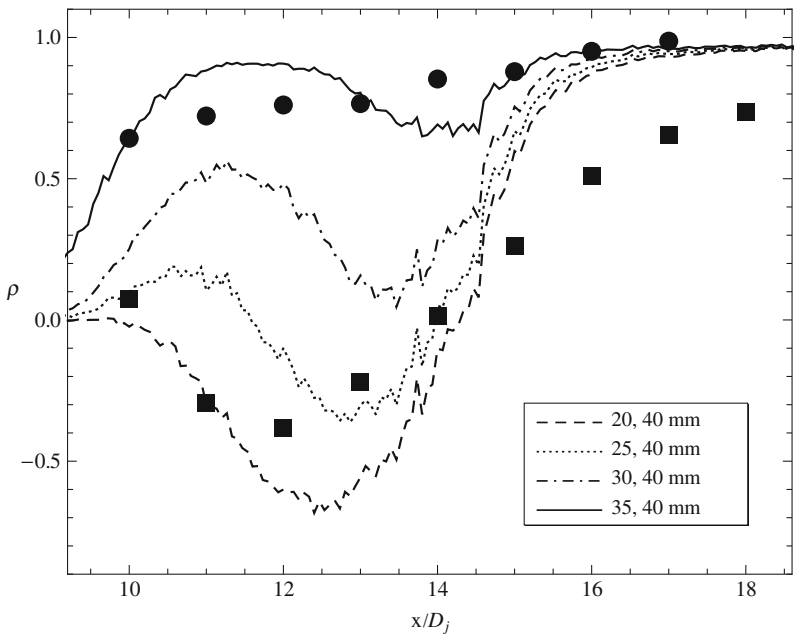


Fig. 9 Transverse profiles of the correlation $\rho$ for the 10,15 $\mathrm{mm}$ ring pair placed at $x_{\text {ring }} / D_{j}=9$ in the turbulent jet. The curves show the simulated profiles with the M2 mesh, while the measured profiles are given for the downstream distances as follows: $\bigcirc, x / D_{j}=10$; $\square, x / D_{j}=11 ; \diamond, x / D_{j}=13$; $\triangle, x / D_{j}=15$. Here, and in Fig. 10, a few measured values that exceed unity have been omitted

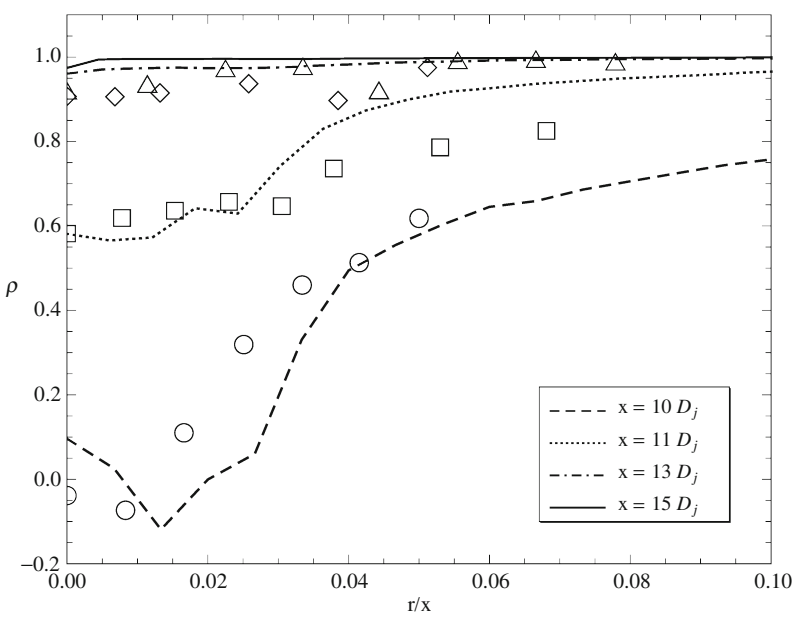

One interesting observation is that the local minimum of $\rho$, while increasing to successively higher $\rho$ values moves radially inward with increasing downstream distance from the sources. In Fig. 10 this trend is clearly indicated by the simulated transverse profiles, but the local minimum is absent from the measured far-field profiles at $x / D_{j}=13$ and $x / D_{j}=15$. The simulations suggest that the local minimum converges to the centerline but the measurements do not give a clear indication as to whether this happens or whether the local minimum disappears prior to arriving at the centerline. As already seen in Figs. 7 and 8, the cross-correlation $\rho$ at $r=0$ for the $35,40 \mathrm{~mm}$ ring pair is not a monotonically increasing function of $x$. Hence, the curves in Fig. 10 are another manifestation of the behavior previously discussed with regard to the centerline dip.

In conclusion, it is seen that LEM3D, despite its limitations, can be a convenient tool for exploring a wider range of parameter space than can be affordably done

Fig. 10 Transverse profiles of the correlation $\rho$ for the 35,40 $\mathrm{mm}$ ring pair placed at $x_{\text {ring }} / D_{j}=9$ in the turbulent jet. The curves show the simulated profiles with the M2 mesh, while the measured profiles are given for the downstream distances as follows: $\bigcirc, x / D_{j}=10$; $\square, x / D_{j}=11 ; \diamond, x / D_{j}=13$; $\triangle, x / D_{j}=15$




experimentally, thereby identifying features that might be suitable targets for subsequent experimental study.

\section{Discussion}

The utility of LEM for subgrid mixing closure of LES is well established. The focus here is on the possible benefits of using LEM to impart spatially and temporally well-resolved unsteadiness to steady-state RANS solutions. This can be done using the splicing strategy previously used for LES closure, as shown in an application of that strategy to RANS mixing closure [10]. Here an alternative subgrid LEM formulation, termed LEM3D, is introduced and its potential benefits have been noted. In the present application, which requires $3 \mathrm{D}$ control volumes much smaller than the integral scale, one advantage is that LEM domains span the entire flow in LEM3D, so eddy events much larger than the control volume edge length can occur (though it is noted that their representation of large-scale 3D motions has some drawbacks). Another advantage is the compatibility of LEM3D with the geometrical structure of ODTLES $[15,16]$, allowing the future possibility of coupling the two methods so that eddy events generated by ODTLES dynamics can be implemented directly in LEM3D. LEM3D can also be used for LES subgrid mixing closure to compare its performance to the LEM splicing strategy.

Apart from these methodological considerations, this study presents the first demonstration of capability to model the challenging mixing experiment of TW. Despite some limitations of model performance largely due to the need for $3 \mathrm{D}$ resolution of the closely spaced scalar sources in that experiment, the measured centerline and transverse two-source cross-correlation profiles are reproduced with good accuracy. The physical fidelity of the model formulation allows assessment of nuances of the associated mixing phenomenology that would be difficult to address for this configuration using a state-space closure model.

In this study, measured flow statistics rather than RANS inputs are used to assign LEM3D parameters. This eliminates the possible impact of RANS errors on the mixing predictions.

LEM3D results are sensitive to numerical implementation details such as the size of the 3D control volumes. Possible improvement in this regard is noted. The sensitivity cannot be thoroughly evaluated here because the chosen application constrains important aspects of numerical implementation. Other such sensitivities have been examined for simple flows by Weydahl [21].

To a large degree, the limitations of LEM3D in this context are the result of reliance on RANS-type steady-state inputs rather than concurrent unsteady time advancement of LEM3D coupled to LES. Though this would undoubtedly improve model performance, as indicated by previous LES-LEM applications, the present goal is to assess the degree of improvement of RANS performance for mixing and combustion applications that can be achieved by introducing LEM closure. Any such improvement would be beneficial due to the relatively low cost and wide usage of RANS. The results presented here suggest that there is at least some significant range of applications for which LEM3D coupling to RANS will provide useful performance improvements. 
Acknowledgements This work forms part of the BIGCO2 project supported by the Research Council of Norway (178004/I30 and 176059/I30) and Gassnova (182070). The authors acknowledge the partners StatoilHydro, ALSTOM, GE Global Research, Statkraft, Aker Kværner, Shell, TOTAL, and ConocoPhillips for their support. The work has also received funding from the European Community's Seventh Framework Programme (FP7/2007-2013) under grant agreement No. 211971 (the DECARBit project).

The work was partially supported by the Division of Chemical Sciences, Geosciences, and Biosciences, Office of Basic Energy Sciences, US Department of Energy. Sandia National Laboratories is a multi- program laboratory operated by Sandia Corporation, a Lockheed Martin Company, for the United States Department of Energy under contract DE-AC04-94-AL85000.

Open Access This article is distributed under the terms of the Creative Commons Attribution License which permits any use, distribution, and reproduction in any medium, provided the original author(s) and the source are credited.

\section{Appendix}

\section{A Random walk by triplet maps}

A random sequence of triplet maps induces a random walk of a given fluid cell, where the fluid cell diff usivity associated with the random walk is interpreted as the turbulent diffusivity $D_{T}$. Thus, following [22], the diffusivity associated with a onedimensional random walk is

$$
D_{T}=\frac{1}{2} R\left\langle\delta^{2}\right\rangle,
$$

where $R$ is the total frequency of displacements of a given fluid cell, and $\left\langle\delta^{2}\right\rangle$ denotes the mean square displacement induced by the events. The frequency of displacements induced by triplet maps of size $3 k d$ is given by $3 k d \lambda f(k)$, where $\lambda$ is the event frequency per unit length and $f(k)$ is the probability of selecting a size $3 k d$ eddy. Here, $d$ is the width of a fluid cell and $3 k$ denotes the number of cells involved in the eddy. The total frequency of displacements of a given fluid cell is expressed as

$$
R=3 \lambda d \sum_{k=k_{\min }}^{k_{\max }} k f(k),
$$

where $k_{\min }$ and $k_{\max }$ are integers determining the smallest and largest eddy, respectively. Similarly, let $\left\langle\delta^{2}(k)\right\rangle$ denote the mean square displacement induced by a single triplet map of size $3 \mathrm{kd}$. The mean square displacement induced by a single eddy event of any size is the average of $\left\langle\delta^{2}(k)\right\rangle$ weighted by the event frequency and by the eddy size $3 k d$ (because the number of affected cells is proportional to the eddy size), so

$$
\left\langle\delta^{2}\right\rangle=\frac{3 \lambda d}{R} \sum_{k=k_{\min }}^{k_{\max }} k f(k)\left\langle\delta^{2}(k)\right\rangle .
$$

We now derive the expression for $\left\langle\delta^{2}(k)\right\rangle$. It can be shown by a direct calculation that the mean square displacement induced by a single eddy of size $3 k d$ is

$$
\left\langle\delta^{2}(k)\right\rangle=\frac{4}{3} k(k-1) d^{2} .
$$


This is obtained by calculating separately the mean square displacements of fluid cells being mapped onto each of the three fluid segments involved in the triplet map, and then adding the contributions to obtain the final result. We here use Eq. 16 as a conjecture in a proof by induction to establish the expression for $\left\langle\delta^{2}(k)\right\rangle$. The induction procedure must be applied twice since triplet maps involving even and odd numbers of cells obey slightly different mapping rules, and hence belong to two different classes. For instance, for a segment consisting of an odd number of cells there is a center cell that is mapped onto itself by a triplet map. Starting with the class of triplet maps involving an even number of cells, the smallest possible triplet map corresponds to $k=2$. This involves six fluid cells, which by the mapping are rearranged from an order 123456 to the order 145236 . Hence, except for the endpoint cells that are mapped onto themselves, all the cells are mapped a distance of 2 cells in either direction in this case. We find that $\left\langle\left.\delta^{2}(k)\right|_{k=2}=4 \cdot(2 d)^{2} / 6=8 d^{2} / 3\right.$, which establishes that the expression (Eq. 16) is true for $k=2$. For the class of triplet maps involving an odd number of cells, the smallest non-trivial mapping corresponds to $k=3$ and involves nine fluid cells. A sequence of cells ' 123456789 ' is rearranged to the order ' 147852369 ' by a triplet map of this size. Thus, in addition to the endpoint cells 1 and 9, the center cell 5 is mapped onto itself. The cells 4 and 6 are mapped a distance of two cells, while the remaining four cells are mapped a distance of four cells in either direction. Therefore, the mean square displacement induced by a triplet map involving 9 fluid cells is $\left\langle\left.\delta^{2}(k)\right|_{k=3}=\left(4 \cdot(4 d)^{2}+2 \cdot(2 d)^{2}\right) / 9=8 d^{2}\right.$. By inspection this shows that the expression (Eq. 16) is true also for $k=3$. We next assume that Eq. 16 holds for an arbitrary integer $k>3$ and show that it is valid for $k+2$. The triplet map of size $3(k+2)$ involves six additional fluid cells compared to the one of size $3 k$. By a direct comparison of the triplet maps involving $3 k$ and $3(k+2)$ fluid cells, it is straightforward to show that two of the additional cells are displaced a distance of $2 k$ cells, while the remaining four additional cells are displaced a distance of $2(k+1)$ cells. This is in fact the case for both classes of triplet maps. The remaining cells involved in the triplet map of size $3(k+2)$ are displaced precisely as the cells involved in a triplet map of size $3 k$. Hence, the mean square displacement induced by a triplet map involving $3(k+2)$ fluid cells becomes

$$
\begin{aligned}
\left\langle\delta^{2}(k+2)\right\rangle & =\left(3 k\left\langle\delta^{2}(k)\right\rangle+4(2(k+1) d)^{2}+2(2 k d)^{2}\right) / 3(k+2) \\
& =\frac{4}{3}(k+2)(k+1) d^{2} .
\end{aligned}
$$

This expression is exactly what is obtained when replacing $k$ with $k+2$ in Eq. 16 . Since it has been shown that Eq. 16 is valid both for $k=2$ and $k=3$, and that the assumption that the expression (Eq. 16) holds for any $k>3$ implies that it holds for $k+2$, this concludes the proof by induction of Eq. 16 .

Inserting Eq. 16 into Eq. 15, and using the relation 13, the turbulent diffusivity becomes

$$
D_{T}=2 \lambda d^{3} \sum_{k=k_{\min }}^{k_{\max }} k^{2}(k-1) f(k) .
$$


B The advection operation

Scalar advective transport in LEM3D, represented by the term $\mathcal{A D} \mathcal{V}$ in Eq. 6, is determined by the mean velocity field $\boldsymbol{U}$ for constant density flows. Thus, for a given time step $\Delta t$, the number of LEM wafers to be displaced across the 3DCV interfaces is $\delta=V_{k} \Delta t / d$, where $V_{k}=3 U_{k}$ is the implemented velocity component normal to a given interface, and $d$ is the wafer thickness. The factor 3 is introduced to compensate for the fact that the LEM wafers on average spend only one third of the time in any one of the coordinate directions. For a general shear flow, the prescribed advective displacements $\delta$ may range from a few percent of a wafer to several wafers. Round-off management is introduced for consistent enforcement of the displacement of an integer number of wafers $\delta$ across the $3 \mathrm{DCV}$ interfaces. The residuals of the round-offs are accumulated and applied whenever they correspond to a whole wafer, thus giving advective wafer displacements that conform to the prescribed transport on average. For example, if the prescribed displacement of fluid across a given interface is $\delta=2.37$ wafers per time step, the actual wafer displacement in general alternates between two and three wafers to give a displacement of 2.37 wafers on average. Displacements at the inlet (upstream) boundary are determined by the round-off management, as are the displacements across the lateral faces of all the 3DCVs. As will be explained in the following, however, the downstream and lateral displacements assigned by the round-off management are preliminary and can be overridden.

In a rigid-body flow, simply shifting the stack of wafers with the flow at every time step would advect the LEM wafers in the streamwise domains. However, if the flow is non-uniform along a given LEM domain, wafers must be transferred into or out of that domain in order to conserve the advected scalars. In LEM3D, transfers of wafers are implemented among the differently oriented LEM domains intersecting each 3DCV, and this provides an advective coupling of the domains. The details of how these wafer transfers take place are governed by the assumption of an incompressible flow and the conservation of mass.

The advective coupling of the LEM domains can be described by designating each domain intersecting a given $3 \mathrm{DCV}$ either as a donor, a receiver, or neutral with respect to that particular $3 \mathrm{DCV}$. A domain is a donor if the flow is compressive in the corresponding direction. In this case the prescribed advection of wafers into and out of the 3DCV for a time step $\Delta t$ causes an excess of LEM wafers in the domain. The excess of wafers must be donated to one or both of the other LEM domains that intersect the $3 \mathrm{DCV}$. In a similar manner, a domain is a receiver if the flow in the corresponding direction is extensional, causing a deficit of LEM wafers in that domain and within the $3 \mathrm{DCV}$ considered. The domain then receives a corresponding number of wafers from one or both of the other intersecting domains, depending on which of these are donors. An LEM domain is neutral with respect to donating or receiving wafers if an equal number of wafers enters and leaves the $3 \mathrm{DCV}$ in the corresponding direction.

With given designations the three LEM domains intersecting a particular 3DCV belong to one out of four classes of cases, categorized by how they distribute in terms of being donors, receivers, or neutral domains. The first class is defined by two of the LEM domains being donors and one domain being a receiver. This makes for three different cases, one for each of the spatial directions being a receiver direction. 
The second class is defined by one of the LEM domains being a donor, while the other two intersecting domains are receivers. This class also includes three different cases, one for each of the spatial directions being a donor direction. The third class is defined by one LEM domain being a donor, another being a receiver, while the third domain is neutral. For this class there are six different cases since for each direction being a donor, the other two directions may interchangeably be a receiver direction or neutral. Finally, the fourth class is defined by all three spatial directions being neutral. This class consists of just one case since there is no difference between the directions in their donor/receiver properties. Altogether, the four classes that the LEM domains intersecting a given 3DCV may fall into constitute a total of 13 different cases. It should be noted that the classification of LEM domains for a given $3 \mathrm{DCV}$ is valid only for one time step $\Delta t$ since the designations of the domains are subject to change for every time step. This is due to the accumulation of non-integer wafer displacements controlled by the round-off management, and in addition a minimization algorithm explained below that further controls the wafer displacements across the 3DCV interfaces.

Conservation of mass is enforced in the global flow domain by requiring the displacements of wafers in and out of each 3DCV to obey the discretized continuity equation

$$
\sum_{l=1}^{N_{\text {faces }}} \delta_{l}=0,
$$

where $\delta_{l}$ denotes the integer number of wafer displacements across the 3DCV faces. The sum on the left-hand-side is over the number of faces of the 3DCV, i.e., $N_{\text {faces }}=6$. The continuity equation is implemented locally in every $3 \mathrm{DCV}$ by the following algorithm. Starting at the upstream boundary of the LEM array structure, the algorithm is initiated by using the prescribed advective displacements to evaluate the integer displacements $\delta_{l}(l=1, \ldots, 5)$ for the upstream 3DCV faces and neighboring lateral faces. Here, the upstream faces are denoted by $l=1$, while the lateral faces of the 3DCVs are denoted by $l=2, \ldots, 5$. The displacements across the downstream faces $(l=6)$ of the upstream first layer of 3DCVs are then calculated from Eq. 19. The obtained values, together with the prescribed displacements of the lateral faces, are used to repeat the calculation for the next layer of 3DCVs, and so on, until the displacements across the downstream faces of the 3DCVs are determined for all the layers. In this manner the assigned integer displacements $\delta_{l}$ across all the 3DCV interfaces are obtained sequentially from the upstream to the downstream boundary of the LEM array structure. The method assumes that the mean flow is streamwise in one of the coordinate directions, and so there is inflow of fluid at the upstream boundary and outflow at the downstream boundary. This current restriction can be relaxed, but it has shown to be useful for the model application presented here because numerical implementation is simplified.

The above outlined algorithm to obey mass conservation generally works well for various flow fields. However, as demonstrated by test cases, the round-offs to integer wafer displacements across the lateral faces may cause occasional cumulative effects which trigger the occurrence of very large deviations from the prescribed streamwise displacements. The occurrence of unacceptably large deviations is prevented by a 
correction algorithm that involves a minimization (subject to a constraint) of the square sum of deviations

$$
d 2=\sum_{l=2}^{N_{\text {faces }}}\left(\varepsilon_{l}+\gamma_{l}\right)^{2},
$$

where the sum is over the 3DCV faces $l$ for which the associated displacements of wafers are subject to change due to the minimization. Thus, the upstream face $(l=1)$ is not included in the above sum since the displacement across this face is already determined either from the upstream boundary conditions (for the first layer of $3 \mathrm{DCVs}$ ), or from the downstream displacement calculated for the 3DCV immediately upstream. For the lateral faces of a given $3 \mathrm{DCV}(l=2, \ldots, 5)$, the quantity $\varepsilon_{l}$ in Eq. 20 represents the difference between the assigned and the prescribed displacement. For the downstream face of the $3 \mathrm{DCV}(l=6), \varepsilon_{l}$ is the difference between the calculated displacement from Eq. 19 and the prescribed displacement. The quantities $\gamma_{l}$ are integer corrections to the preliminary assigned displacements, obtained by minimizing $d 2$ and subject to the constraint $\sum \gamma_{l}=0$ to preserve continuity. By the minimization algorithm, final values of the displacements across all interfaces of the 3DCV are determined. Since neighboring 3DCVs share a common face, the minimization of $d_{2}$ is only feasible, but also sufficient, for the 3DCVs belonging to one of the two checkerboard subsets of the set of all 3DCVs in each layer. To ensure a consistent procedure, the selected checkerboard subset is alternated for each successive layer of $3 \mathrm{DCVs}$, and also for successive timeadvancement cycles.

From the above it follows that at every advective time step $\Delta t$ the conservation of mass implies the transfer of LEM wafers from the donor domain(s) intersecting a given $3 \mathrm{DCV}$ to the receiver domain(s) of that same 3DCV. The continuity Eq. 19 ensures that the excess of wafers in the donor(s) exactly matches the deficit of wafers in the receiver(s). The transfer of wafers between the LEM domains is implemented in such a way that wafers to be donated are extracted from the center of the $3 \mathrm{DCV}$, flipped, and then inserted into the center of the same 3DCV in the receiver domain(s). The choice of extracting wafers from and inserting them into the 3DCV centers minimizes artificial transport by the flipping process. The insertion of donor segments into the receiver(s) furthermore involves an arbitrariness in terms of the ordering of the wafers. In LEM3D, the possible options for the ordering of inserted wafers are randomized and given equal probabilities to prevent any biases.

To illustrate the advection operation when flipping of wafers occurs and the associated randomness, let us assume that the intersecting LEM domains of a given 3DCV belong to the third class of donor-receiver cases, consisting of one donor, one receiver, and one neutral domain. In Fig. 11 is shown the flipping and advection of two excess wafers from a donor into a receiver for this case. The two wafers extracted from the center of the donor leave a gap which is filled by the advection of wafers in that domain. In an opposite manner, the advection of wafers in the receiver leaves vacancies that are filled by the flipped wafers when inserted. Introducing a notation for the flipping process, the wafers to be donated are extracted from a fluid interval $\left[d_{1}, d_{2}\right]$ of the donor and transferred to an equally sized fluid interval $\left[r_{1}, r_{2}\right]$ of the receiver. With two or more wafers to be transferred from the donor to the receiver, a wafer segment such as $\left[d_{1}, d_{2}\right]$ can be inserted into the receiver in two different ways. Thus, by a re-ordering, the wafer segment can be transferred to the interval $\left[r_{1}, r_{2}\right]$ 

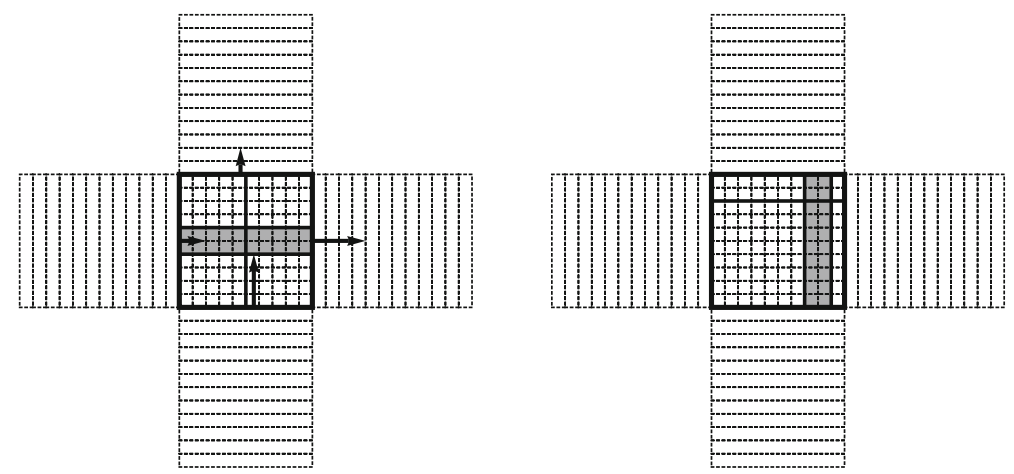

Fig. 11 A 2D illustration of the advection operation associated with a given 3DCV for the third class of donor-receiver cases. The arrows in the left figure indicate the given wafer displacements in the donor domain (vertical) and the receiver domain (horizontal), with the resulting displacements shown in the figure to the right. The thin solid vertical line through the 3DCV center on the left cuts the receiver into two parts, with the wafer displacements on either side leaving a gap as indicated by the two thin solid vertical lines in the right figure. The two excess wafers (in gray) in the donor are extracted from the center of the 3DCV, rotated clockwise or counterclockwise (randomly chosen), and then displaced and inserted into the receiver so as to fill the gap created by the advection of that domain. The wafer displacements in the donor correspondingly fill the gap left by the extracted wafers, indicated by the merging of the two thin solid horizontal lines in the left figure into the single thin solid horizontal line in the right figure. The neutral domain is normal to the paper plane and not shown in the illustration

either in the order $\left[d_{1}, d_{2}\right]$ or in the order $\left[d_{2}, d_{1}\right]$. These two options are randomized and given equal probabilities to prevent biases.

Similar randomization is implemented within the classes of donor-receiver cases defined either by two donors and one receiver, or one donor and two receivers. Thus, for the first class involving two donors, the receiver will receive LEM wafers from two different sources $\left[d_{1}, d_{2}\right]$ and $\left[d_{3}, d_{4}\right]$, under the assumption that both donors contain a surplus of two wafers. With no obvious physical guidelines concerning the ordering of the donor segments themselves, the insertion is randomized with equal probabilities given to the two possible segment orderings. In addition, if the donor segments contain two or more wafers, the ordering of the wafers for each of the segments upon insertion is randomized as discussed above for the third class of donor-receiver cases. Hence, if both donor segments contain a multiple number of wafers, there are a total of eight different ways of performing the transfer of excess wafers from the two donors to the receiver. All of these eight options are implemented with equal probabilities. For the second class of donor-receiver cases, there are similarly eight different ways, all with equal probabilities, of transferring the excess wafers from the one donor to the two receivers. It should be noted that wafers belonging to a given donor segment neither are mixed internally nor with wafers from another donor segment by the flipping and insertion algorithm.

\section{Auxiliary coupling between LEM domains}

The advection operation $\mathcal{A D} \mathcal{V}$ with transfer of wafers among the differently oriented LEM domains, as described in Appendix B, in general provides a coupling between these domains. However, in order to ensure that physical processes are consistently 
represented in all spatial directions, an auxiliary coupling of the arrays of mutually orthogonal LEM domains must be incorporated. This is motivated by considering for instance a uniform streamwise flow and homogeneous turbulence. In this case a scalar injected into the streamwise oriented LEM domain will never spread into the laterally oriented domains unless the domains are coupled by an additional process. Hence, the auxiliary coupling is needed to alleviate the possible insufficiency of LEM domain coupling in certain regions of a flow, and thus to assure that the simulation algorithm produces three correlated realizations of the scalar fields.

The auxiliary coupling, represented by the operation $\mathcal{A C}$ in Eq. 6, is implemented by stochastic rotations of the $3 \mathrm{DCV}$. The rotations give additional fluid exchanges between the LEM domains, compared to those implied by the operation $\mathcal{A D} \mathcal{V}$. Thus, for every global time step $\Delta t$, the $3 \mathrm{DCVs}$ are rotated $\pm 90^{\circ}$ about any one of the three coordinate axes with a locally defined probability

$$
p_{\text {rot }}=\frac{3 \mathrm{CFL} V_{3 \mathrm{DCV}}}{2 V_{\max }}
$$

where CFL is the Courant-Friedrichs-Lewy number, $V_{3 \mathrm{DCV}}$ is the absolute value of the largest velocity component associated with the 3DCV side faces, and $V_{\max }$ is the maximum $V_{3 \mathrm{DCV}}$ of the computational domain. This gives six different ways of rotating a $3 \mathrm{DCV}$, all with an equal probability of being performed if a rotation is to occur. For the particular $3 \mathrm{DCV}$ for which $V_{3 \mathrm{DCV}}=V_{\max }$, the rotation probablility is $p_{\text {rot }}=\frac{3}{2}$ CFL. The origin of the factor $\frac{3}{2}$ is explained below and implies that the implementation allows for a maximum CFL number given by $\mathrm{CFL} \leq \frac{2}{3}$.

The $\pm 90^{\circ}$ rotation of a $3 \mathrm{DCV}$ gives exchange of fluid elements between the LEM domains orthogonal to the rotational axis. Fluid elements in the LEM domain along the rotational axis, however, are unaffected by the rotation. Thus, $\frac{2}{3}$ of the LEM wafers in a $3 \mathrm{DCV}$ are affected by a rotation while the remaining $\frac{1}{3}$ are not affected. The factor $\frac{3}{2}$ in Eq. 21 implies that on average the individual 3DCVs are rotated 1.5 times for every time period

$$
\tau_{\mathcal{A D V}}=M / V_{3 \mathrm{DCV}}
$$

corresponding to the advective residence time of the LEM wafers within the 3DCVs. Therefore, on average, all LEM wafers within a given $3 \mathrm{DCV}$ will be displaced to differently oriented domains over the time scale $\tau_{\mathcal{A D V} \mathcal{V}}$.

One feature of the auxiliary coupling is that the $3 \mathrm{DCV}$ rotations introduce additional transport of the wafers. This transport is not directly physically motivated, but the extra transport can be evaluated and counterbalanced by deducting the corresponding diffusivity from the turbulent diffusivity $D_{T}$. Thus, using that the rate of $3 \mathrm{DCV}$ rotations is $R=3 /\left(2 \tau_{\mathcal{A D V} \mathcal{V}}\right)$, we get from Eqs. 13 and 22 that the diff usivity associated with rotations of a given $3 \mathrm{DCV}$ is

$$
D_{T}^{\mathrm{rot}}=\frac{3 V_{3 \mathrm{DCV}}}{4 M}\left\langle\delta^{2}\right\rangle
$$

where $\left\langle\delta^{2}\right\rangle$ is the mean square single rotation displacement of the wafers in the 3DCV in a given direction orthogonal to the rotational axis. Taking into account that only 
$\frac{2}{3}$ of the wafers are affected by a rotation, the mean square displacement due to a single rotation can be expressed as

$$
\left\langle\delta^{2}\right\rangle=\frac{2}{3} \sum_{k=1}^{n / 2} \frac{((2 k-1) M / 2 n)^{2}}{n / 2},
$$

where $n$ is the number of wafers per $3 \mathrm{DCV}$ in any of the coordinate directions. From this, the diffusivity associated with rotations of the $3 \mathrm{DCVs}$ is

$$
D_{T}^{\mathrm{rot}}=\frac{M V_{3 \mathrm{DCV}}}{4} \sum_{k=1}^{n / 2} \frac{(2 k-1)^{2}}{n^{3}} .
$$

The expression $D_{T}^{\text {rot }}$ is subtracted from the turbulent diffusivity $D_{T}$ in the LEM3D implementation. This has the effect that a fraction of the turbulent transport implemented by the triplet maps is replaced by the 3DCV rotations. In the outer regions of the flow domain, the fraction may exceed unity, with the implication that the rotation-induced transport exceeds the physically specified transport that it replaces (a model artifact). It should be noted, however, that the effect of the excess transport is negligible in this application and should be negligible more generally.

Another coupling artifact of the model is that the 3DCV rotations bring dissimilar fluid states into contact. A remedy to this issue is to use coarser $3 \mathrm{DCV}$ s to minimize the artifact. Such an approach also has the benefit of being computationally less expensive than using smaller 3DCVs for given spatial resolution of the LEM wafers because it reduces the total number of wafers in the simulation. The computational cost saving of using larger 3DCVs must be balanced by the model performance of LEM3D simulations, however, since other model artifacts are likely to become strong if the 3DCVs are too large. In particular, the coarse-scale 3D-resolution must under any circumstance be fine enough so that mean-flow resolution requirements are fulfilled.

\section{References}

1. Kerstein, A.R.: A linear-eddy model of turbulent scalar transport and mixing. Combust. Sci. Technol. 60, 391-421 (1988)

2. Kerstein, A.R.: Linear-eddy modelling of turbulent transport, part 6. Microstructure of diffusive scalar mixing fields. J. Fluid Mech. 231, 361-394 (1991)

3. Kerstein, A.R.: Linear-eddy modelling of turbulent transport, part 7. Finite-rate chemistry and multi-stream mixing. J. Fluid Mech. 240, 289-313 (1992)

4. McMurtry, P.A., Gansauge, T.C., Kerstein, A.R., Krueger, S.K.: Linear eddy simulations of mixing in a homogeneous turbulent flow. Phys. Fluids A 5, 1023-1034 (1993)

5. McMurtry, P.A., Menon, S., Kerstein, A.R.: A linear eddy sub-grid model for turbulent reacting flows: Application to hydrogen-air combustion. Proc. Combust. Inst. 24, 271-278 (1992)

6. McMurtry, P.A., Menon, S., Kerstein, A.R.: Linear eddy modeling of turbulent combustion. Energy Fuels 7, 817-826 (1993)

7. Menon, S,. Calhoon, J.W.H.: Subgrid mixing and molecular transport modeling in a reacting shear layer. Proc. Combust. Inst. 26, 59-66 (1996)

8. Chakravarthy, V.K., Menon, S.: Subgrid modeling of turbulent premixed flames in the flamelet regime. Flow Turbul. Combust. 65, 133-161 (2000)

9. Sankaran, V., Menon, S.: Subgrid combustion modeling of 3-D premixed flames in the thinreaction-zone regime. Proc. Combust. Inst. 30, 575-582 (2005)

10. Goldin, G.M., Menon, S., Calhoon, J.W.H.: A linear eddy mixing model for steady non-premixed turbulent combustion. AIAA Pap. (95-0379) (1995) 
11. Tong, C., Warhaft, Z.: Passive scalar dispersion and mixing in a turbulent jet. J. Fluid Mech. 292, 1-38 (1995)

12. Warhaft, Z.: The interference of thermal fields from line sources in grid turbulence. J. Fluid Mech. 144, 363-387 (1984)

13. Tennekes, H., Lumley, J.L.: A First Course in Turbulence. The MIT Press, Cambridge, MA (1972)

14. Menon, S., Kerstein, A.R.: The Linear-Eddy model. In: Echekki, T., Mastorakos, E. (eds.) Turbulent Combustion Modeling: Advances, New Trends, and Perspectives, pp. 221-247. Springer, Dordrecht, Germany (2011)

15. Schmidt, R.C., Kerstein, A.R., McDermott, R.: ODTLES: a multi-scale model for 3D turbulent flow based on one-dimensional turbulence modeling. Comput. Methods Appl. Mech. Eng. 199, $865-890$ (2010)

16. Gonzalez-Juez, E.D., Schmidt, R.C., Kerstein, A.R.: ODTLES simulations in wall-bounded flows. Phys. Fluids 23, 125102 (2011)

17. Kerstein, A.R., Ashurst, W.T., Wunsch, S., Nilsen, V.: One-dimensional turbulence: vector formulation and application to free shear flows. J. Fluid Mech. 447, 85-109 (2001)

18. Viswanathan, S., Pope, S.B.: Turbulent dispersion from line sources in grid turbulence. Phys. Fluids 20, 101514 (2008)

19. Panchapakesan, N.R., Lumley, J.L.: Turbulence measurements in axisymmetric jets of air and helium, Part 1. Air jet. J. Fluid Mech. 246, 197-223 (1993)

20. Wolfram Research Inc: Mathematica, Version 7.0. Wolfram Research, Inc., Champaign, IL (2008)

21. Weydahl, T.: A framework for mixing-reaction closure with the Linear Eddy Model. PhD thesis, NTNU Trondheim, Norwegian University of Science and Technology, Department of Energy and Process Engineering. (2010)

22. Chandrasekhar, S.: Stochastic problems in physics and astronomy. Rev. Mod. Phys. 15, 1-89 (1943) 